\title{
Development Trend and Application of Star Soil Sampler Impact Mechanism Technology
}

\author{
Lu Mingyu, Zhang Ming, Liu Shuo, Zhang Xingwang
}

China Academy of Space Technology, Beijing Spacecraft, Beijing, China

\section{Email address:}

lumingyu@126.com (Lu Mingyu)

\section{To cite this article:}

Lu Mingyu, Zhang Ming, Liu Shuo, Zhang Xingwang. Development Trend and Application of Star Soil Sampler Impact Mechanism Technology. Science Discovery. Vol. 7, No. 1, 2019, pp. 1-8. doi: 10.11648/j.sd.20190701.11

Received: December 6, 2018; Accepted: January 24, 2019; Published: February 1, 2019

\begin{abstract}
With the development of global space technology, technologies of planet soil detection, sampler and return will become more and more important in the field of deep space exploration in the future. The space environment detection and sampling tasks in deep space exploration have the characteristics of high vacuum, low gravity, intense temperature change, complex composition of target materials, and limited energy and power consumption of the sampler. Therefore, in the extremely harsh space environment, the ability of the sampler to break the surface material of the celestial body directly determines the completion of the sampling task. In view of the above situation, the introduction of impact excitation in the extraterrestrial celestial sampling returning device can improve the intrusion ability of the sampling device to hard materials, and can effectively reduce the static pressure and total energy required during the drilling process, and the vibration brought by the drilling device is also It is beneficial to chip removal and troubleshooting such as card drilling, which can effectively improve the drilling and crushing efficiency of drilling and mining equipment. Therefore, impact sampling is an ideal sampling method for deep space detection.The characteristic and rock breaking mechanism of impact load are analyzed by this paper, the application status and development trend of impact drilling space sampler were investigated, the research direction and suggestion of the impact mechanism of planet soil sampler are put forward.
\end{abstract}

Keywords: Deep Space Exploration, Planet Soil Sampling, Impact Mechanism, Fracture Mechanism, Energy Transfer Efficiency

\section{星壤采样器冲击机构技术发展趋势与应用}

\author{
路明雨, 张明, 刘硕, 张兴旺 \\ 中国空间技术研究院, 北京卫星制造厂有限公司, 北京, 中国
}

\section{邮箱:}

lumingyu@126.com(路明雨)

\begin{abstract}
摘要: 随着全球航天技术的发展, 星壤探测及采样返回技术在未来深空探测领域的作用将会愈发重要。深空探测中的空 间环境探测采样任务具有高真空、低重力、温度变化剧烈、目标物质成分构成复杂, 采样器搭载的能源和功耗有限等特 点。因此, 在极端恶劣的空间环境条件下, 采样器对地外天体表面物质的破碎能力直接决定了采样任务的完成情况。针 对以上情况, 在地外天体采样返回设备中引入冲击激励作用能够提高采样设备对坚硬物质的侵入能力, 可以有效减小钻 进过程中所需要的静压力和总能量, 其带来的振动也有利于排屑和排除卡钻等故障, 能够有效提高钻采设备的钻进碎岩 效率。因此, 冲击式取样是一种较为理想的深空探测采样方式。文章分析了冲击载荷的特点和碎岩机理, 在调研国内外 冲击钻进式航天采样器应用现状和发展趋势的基础上, 提出了我国星壤采样器冲击机构的研究方向和建议。
\end{abstract}


关键词: 深空探测, 星壤采样, 冲击机构, 破碎机理, 能量传递效率

\section{1. 引言}

随着“绕、落、回”探月三步走任务即将完成, 我国已 开始规划后续深空探测任务计划, 地外天体钻取采样返回 探测技术作为重要的接触式探测手段, 是研究星体表面可 利用资源的重要途径, 也是需要重点强化和发展的专业方 向。由于地外天体表面为微重力环境, 同时星壤中存在不 同粒径, 不同硬度等级的岩石, 还可能存在水冰和冻土等 物质。为了弥补可用预压力的不足, 提高采样器对坚硬物 质的破碎效果, 地外天体探测采样过程中需要引入冲击激 励作用。1970年-1976年间, 前苏联发射的Luna系列和 1969年－1972年间美国发射的阿波罗系列月球探测器，均 采用了搭载冲击机构的采样设备, 通过施加冲击载荷的方 式实现了钻取采样目标 [1]。近年来, 人们的地外天体探测 由月球逐渐向火星、小行星、彗星等更远的目标发展, 其 中包括英国发射的“Beagle-2”火星探测器、美国发射的 “Spirit”火星探测器（MER-A）、NASA LSAS火星表面采 样器以及欧空局执行的“Rosetta”彗星探测计划等。这些探 测活动中所使用的钻取采样装置几乎全部采用“冲击+回 转” 的工作模式, 已经取得了一定的进展。以上的采样机 构大部分是通过激励装置驱动储能弹簧, 将弹簧的弹性势 能转化为冲锤机构的动能, 使其与钻具发生碰撞以实现冲 击作用进而提高采样器对星壤及星岩物质的破碎潜入能 力。这对我国未来深空探测任务中钻取采样设备的研制具 有重要的借鉴意义。冲击作用不仅能降低介质的抗破坏能 力, 提高设备对坚硬物质的破碎效率, 其带来的振动也有 利于排屑和排除卡钻等故障, 并且冲击作用产生的摩擦和 切削热量较少, 不会对低温环境中的样品产生影响, 能够 保持样品原始信息与层理特性。对研究地外天体表层及次 表层物质成分、机械物理特性及地质组构具有重要意义, 是地外天体表面采样较为理想的方式, 可作为主要研究方 向, 高效的冲击机构与采样方案将作为后续关键技术攻关 重点。

目前, 哈尔滨工业大学已经对地面上应用的冲击钻进 机构中应力波的传递机理以及冲击频率和单次冲击功等条 件对碎岩效果的影响展开了研究; 南京航空航天大学也开 展了针对高频冲击载荷驱动装置在航天器采样设备中应用 的探索。虽然这些研究已经取得了一定的成果, 但对于冲 击机构本身的锤杆质量匹配关系、材料刚度设计、钻具构 型设计等关键因素对冲击波能量在钻具中的传递过程和其 对岩石破碎效果的影响进行的研究相对比较贵乏。同时, 针对高真空低重力空间环境下的探测器采样任务, 如何有 效的以冲击惯性力替代静压力, 提高采样器对坚硬介质的 破碎能力和效率依然是国内外深空探测及其相关领域需要 研究的技术重点。因此, 对航天采样器冲击机构进行调研,
开展冲击载荷传递机理和工作时的力学特性研究具有十分 重要的意义和价值。

\section{2. 冲击作用的产生和碎岩机理}

\section{1. 冲击机械系统简介}

冲击机械利用碰撞作用而产生强度极大的力流, 与相 同功率下的静压机械相比, 其结构更为紧凑, 在工业生产 中有着广泛的应用。在矿山开采中有丵岩机、潜孔钻、风 镐和碎石器等; 在土建工程中有打桩锤、夯实锤和射钉机; 在机械加工行业中有锻锤、冲床、铆钉机以及在后文中提 到的航天器采样机构[2]。虽然冲击机械适用的场合和使用 动力不同, 内部结构也有较大的差异, 但他们都有着相同 的动作原理: 冲击机械中的冲锤在重力、液压、或电机驱 动的作用下加速运动, 并以一定的速度撞击工作对象, 或 通过中介物间接撞击工作对象，使得工作对象发生位移、 变形和破坏。然后, 冲锤又在机械力、液压、蒸汽和燃气 等力的作用下做回程运动, 如此往复, 实现冲击机械的预 定功能[3]。

冲击载荷作用下破碎介质是钻进过程中常见的一种方 式, 现有的钻探装置如电钻、冲击钻、电锤等, 它们的工 作原理都是利用传动机构在静止或带动钻头做旋转运动的 同时, 施加一个方向垂直于钻头的往复锤击运动, 致使被 钻介质破碎[4]。

\section{2. 冲击作用碎岩机理}

冲击钻进与回转钻进不同, 回转钻进时, 钻头在轴 向静载荷（即钻压）的作用下, 吃入岩石一定深度。同 时在回转作用下, 钻杆以吃入岩石深度为“进刀量”连续地 切削岩石。而在冲击载荷作用下, 钻头在钻压力的作用 下, 给孔底岩石一预压应力, 在此预压应力的作用下, 钻头并不直接侵入岩石, 只是使其下区域的岩石产生弹 性变形。在冲击力的作用下, 由于能量的急剧集聚, 已 经产生弹性变形的岩石会产生体积破碎坑[5]。同时随着 时间的推移可以将破碎坑扩大, 并将冲击钻头变换位置。 当下一次的冲击应力波到来时, 该钻杆又会在新的位置 破碎岩石[6]。

当冲击功通过钻头传递到孔底岩石上时, 会在与钻孔 轴线平行的面内剪切型裂纹以及位于钻孔轴线和直径构成 的平面上裂纹的端部引发高密度的应力集中, 迅速将微型 裂纹扩展, 使其成为宏观裂纹, 并将已形成的宏观裂纹撕 裂, 在孔底岩石表面形成破碎小坑。同时, 未被消耗掉的 冲击功以应力波的形式继续向下传播, 使已有微观裂纹张 口、扩展、连通、甚至断裂, 并萌生新的微观裂纹。冲击 功越大, 则破碎小坑越大、微观裂纹的扩展深度越深、萌 生数量越多, 越有利于岩石的破碎[7]。 


\section{3. 航天器采样器冲击机构研究现状}

\section{1. 上世纪美苏探月冲击式采样设备}

从上世纪五十年代以来, 前苏联和美国共向月球发射了近百颗月球探测器, 采集到的月壤和岩石样品总计约 $382 \mathrm{~kg}[8]$ 。 前苏联在1970年至1976年间共成功进行了3次月面无人自动采样返回任务。三次采样任务技术指标见表1。

表1 前苏联月面采样技术指标。

\begin{tabular}{llll}
\hline 任务 & Luna16 & Luna20 & Luna24 \\
\hline 钻具尺寸 $(\mathrm{mm})$ & $690 \times 290$ & $690 \times 290$ & 约3000×500×500 \\
钻具功率 $(\mathrm{W})$ & 140 & 140 & 900 \\
采样深度 $(\mathrm{mm})$ & 350 & 250 & 1600 \\
采样质量 $(\mathrm{g})$ & 101 & 55 & 170 \\
样品成分 & 玄武岩质碎屑、熔融玻璃状结构碎屑 & 斜长岩碎片 & 分层的细粒月壤和月尘, 长石颗粒 \\
备注 & $/$ & 出现卡钻、提钻困难情况 & $/$ \\
\hline
\end{tabular}

如图1所示, Luna16/20探测器采用摆杆式钻探采样装置, 安装在机械臂上的钻探采样装置通过机械臂的摆动, 实现 月面钻探采样和样品封装 $[9,10]$ 。

在Luna20探测器采样过程中, 三次出现钻进负载达到最 大极限的情况 [11], 在钻孔深度为 $40 \mathrm{~mm} 、 100 \mathrm{~mm}$ 和 $150 \mathrm{~mm}$ 时可能出现了卡钻、驱动电机跳闸、停钻等现象。按照故障 处理预案启动冲击作用后, 钻取采样装置突破了“致命”的困 难孔段, 钻机在 $100^{\circ} \mathrm{C}$ 高温的月表条件下继续钻进。采样任 务结束时, 钻进深度为 $290 \mathrm{~mm}$, 返回地球的月壤约为 $55 \mathrm{~g}$ [12]。

如图2所示，1976年8月发射的Luna24探测器采用了滑轨 式钻取采样装置。采样钻具安装在两个导轨之间, 在进尺驱 动机构的作用下沿着滑轨向下钻进。Luna24的钻取采样装置 总重量为 $54 \mathrm{~kg}$, 包含回转钻进和回转冲击钻进两种工作模式。 依据在线监测到的不同钻压力, 钻具驱动装置可以在两种工 作模式之间自主切换[13]。当钻进到一定深度时, 由于钻进 阻力急剧增大, 钻取采样装置多次自动启动了冲击作用。最 终钻探深度达到 $2.25 \mathrm{~m}$, 取样长度为 $1.6 \mathrm{~m}$, 采样质量为 $170 \mathrm{~g}$ 。

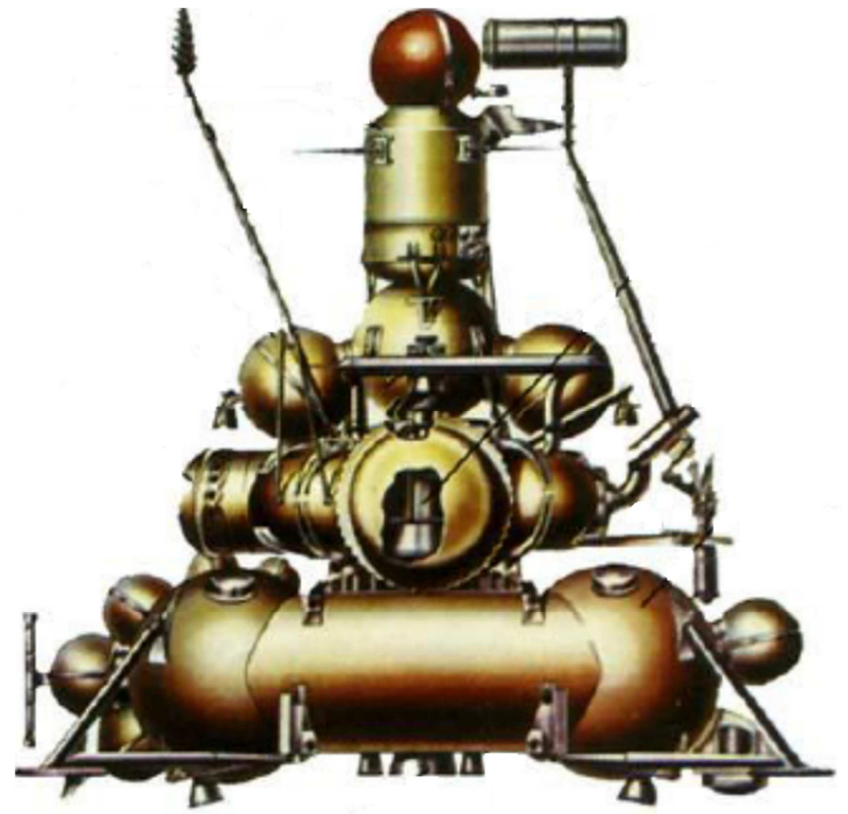

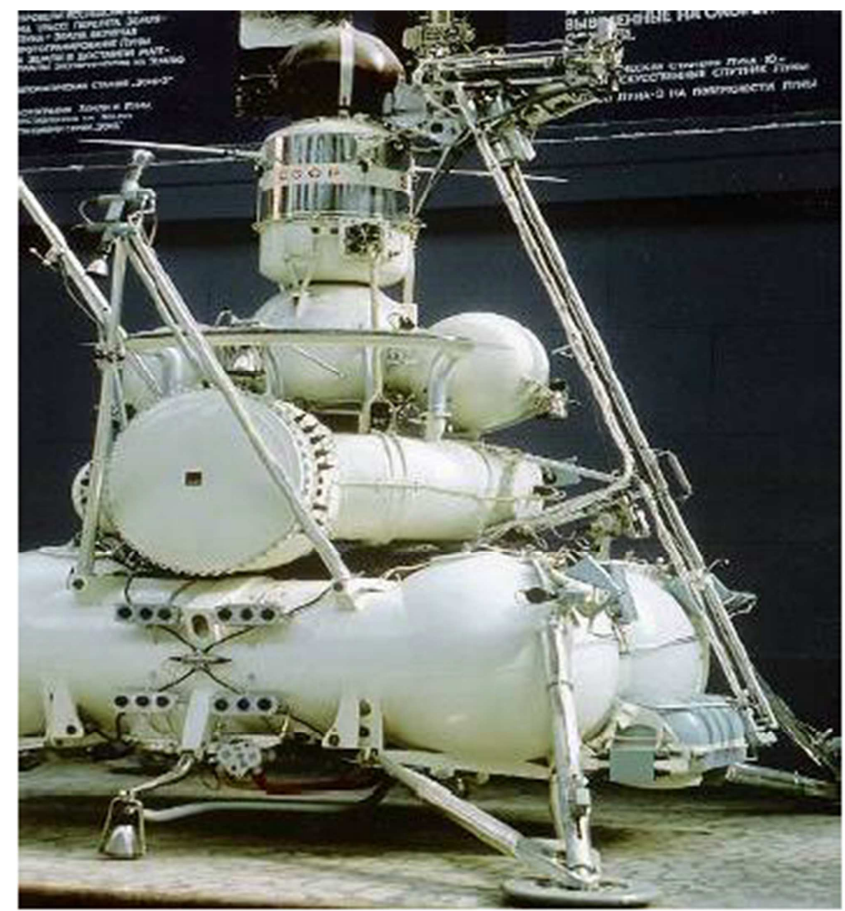

图2 Luna24探测器。

综上所述, 在前苏联的Luna系列探测器中, 冲击机构 的作用主要是在出现卡钻、驱动电机跳闸、停钻等现象时, 通过冲击作用来排除这些故障以继续钻进过程。

美国是世界上开展地外天体采样任务次数最多的国家, 从二十世纪六十年代的Apollo系列月球探测器到火星探测 计划, 将近半个世纪的时间里, 美国积累了大量的宝贵经 验和技术资料, 对我国的探月工程的实施具有很重要的参 考价值。

美国月面采样任务主要是通过载人登月的方式实现。 在1969年到1972年之间, 美国共六次发射Apollo系列探测器, 其中Apollo-15、16、17均采用表取和钻取相结合的取样方 式。Apollo系列探测器的钻取采样方式和采样钻具如图3所 示。

图1 Luna16/20探测器。 


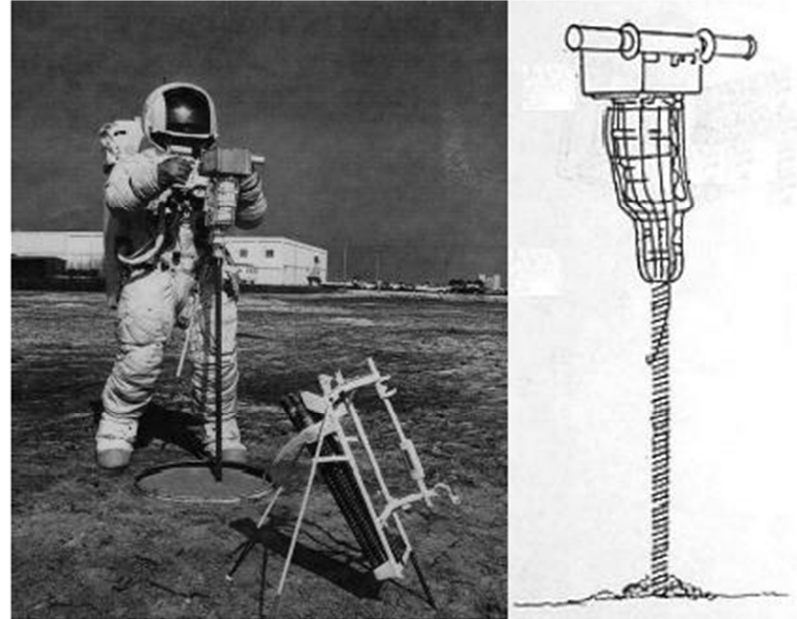

图3 Apollo探测器钻取采样工具。

Apollo系列探测器所采用的采样装置为手持式回转冲击 钻, 冲击作用的实现方式为: 电机通过端面凸轮, 将回转运 动转化为激振块的轴向运动; 激振块在储能弹簧的作用下与 钻杆发生碰撞, 最终实现钻杆的轴向冲击; 冲击功的大小由 激振弹簧刚度和冲击振幅（弹簧压缩量）决定。冲击机构起 到的作用主要是增强钻具潜入月壤的能力，以冲击作用和回 转作用耦合的方式提高钻具破碎月壤和岩石的效率。

Apollo-15探测器钻取采样装置的回转冲击参数如表2所示 [14]。

表2 Apollo-15探测器钻取采样装置回转冲击参数。

\begin{tabular}{llll}
\hline 名称 & 数值 & 名称 & 数值 \\
\hline 钻机功耗 $(\mathrm{W})$ & 450 & 钻杆转速 $(\mathrm{r} / \mathrm{min})$ & 202 \\
钻杆转矩 $(\mathrm{N} \cdot \mathrm{m})$ & 14.7 & 取样直径 $(\mathrm{mm})$ & 19.1 \\
钻进速率 $(\mathrm{mm} / \mathrm{min})$ & $13 \sim 40$ & 激振频率 $(\mathrm{Hz})$ & 37.5 \\
单次冲击功 $(\mathrm{J})$ & 3.4 & 激振块质量 $(\mathrm{g})$ & 299 \\
冲击振幅 $(\mathrm{mm})$ & 12.7 & 弹簧刚度 $(\mathrm{N} / \mathrm{m})$ & 41993 \\
\hline
\end{tabular}

\section{2. 深空探测采样器冲击机构研究进展}

2011年11月26日，美国NASA发射了一颗名为“好奇号” 的火星探测器 [15]。“好奇号”携带有火星土壤采样装置, 用 于采集火星土壤和火星岩石并进行就位检测分析，判断火 星的地理环境条件[10]。

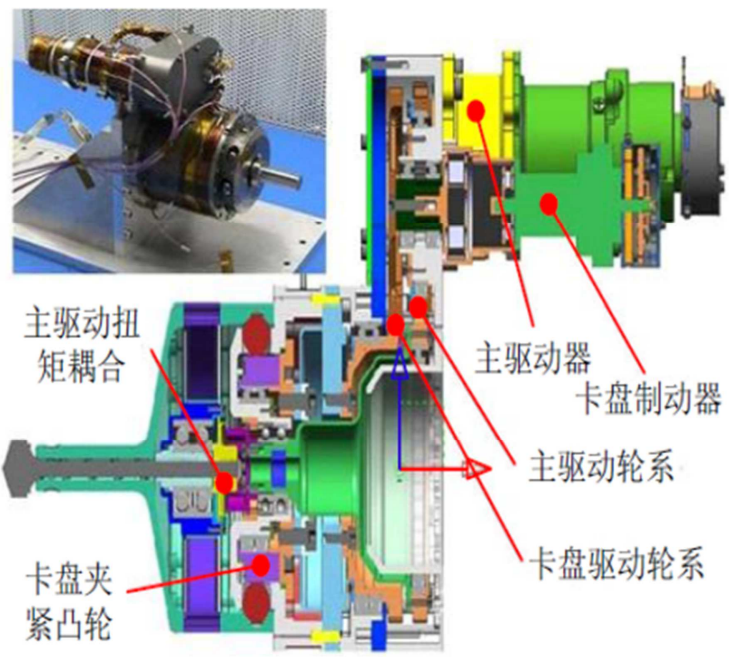

(a)钻具驱动机构

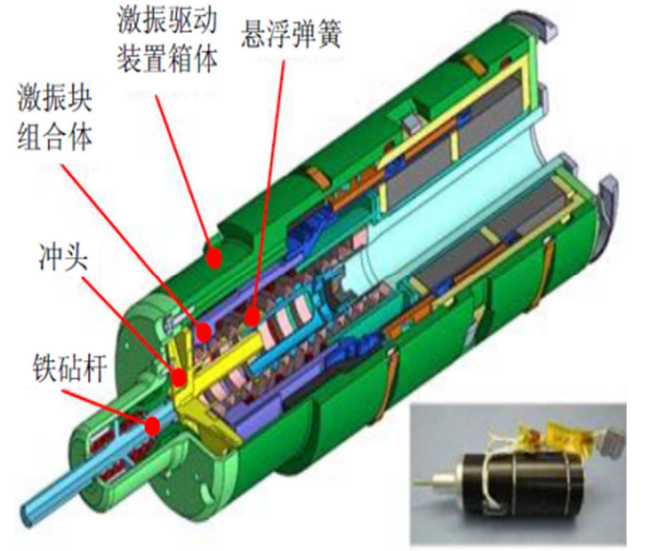

(b)激振驱动机构

图4 “好奇号”火星探测器钻取采样装置。

如图4 (a) 所示, “好奇号”火星探测器的采样钻具驱动 装置包括回转驱动机构和冲击激励驱动机构两部分。冲击 激励驱动机构的作用是为钻具提供破碎岩石的冲击能量和 为粉末状样品提供转送所需要的振动环境。图4 (b) 所示, 激振驱动机构通过压缩弹簧储能驱动激振块敲击钻杆尾部, 实现冲击作用, 其中激振块质量为 $0.4 \mathrm{~kg}$, 激振频率最高为 $30 \mathrm{~Hz}$, 单次冲击功在0.05-0.8J的范围内连续可调 $[16,17]$ 。

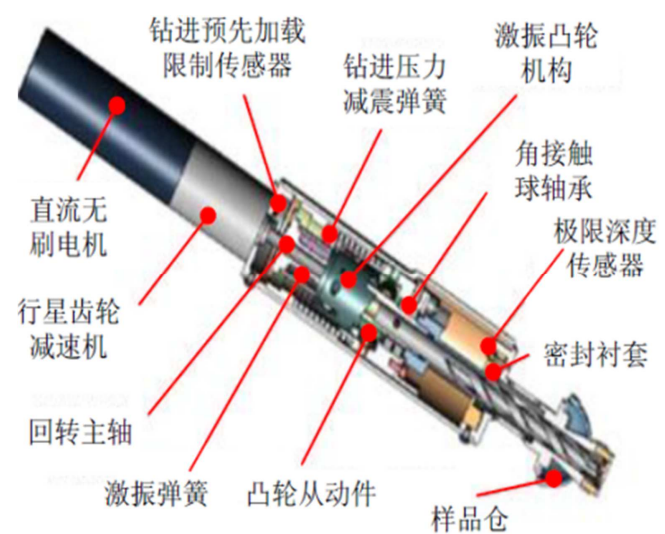

(a) LSAS浅层采样器

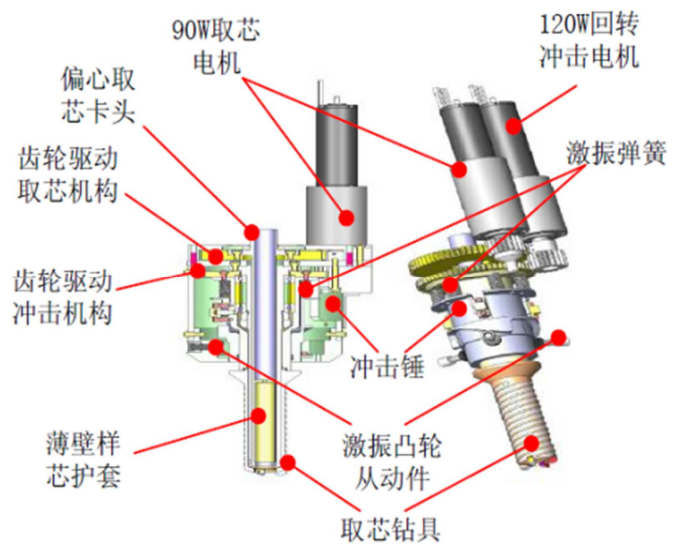

(b) LSAS岩心采样钻机

图5 LSAS采样机构内部结构。 
2006年, NASA投资研发了一种LSAS浅层采样器, 用 于其火星装备研发项目 (MIDP), 采样器的结构如图5 (a) 所示。LSAS原理样机模型采用单电机驱动, 其工作模式包 括冲击钻进和回转钻进 [18]。

(LSAS) 是由Alliance开发的具有集成采样能力的冲击 钻。在轻型平台的支持下, 打算钻入各种各样的岩石和冻 土, 主要设计目标是尽量减少钻进时的作用力以及钻采机 构的质量, 体积, 包络和功率 (Stanley, Dougherty and Laramee，2007）。这个简单而有效的机构使用单电机通过 钻进目标材料的表面获得样品。为确保寿命和避免污染问 题, 钻头采用非常特殊的材料制成, 且采用适用于太空的 无刷直流电机驱动。钻机动作是锤式驱动, 可以使机构以 最小的力量获取样品。重锤由马达驱动的凸轮和弹簧/自由 质量系统驱动。当样品从岩石表面或孔中脱落时, 可以通 过钻头上的槽被进入到存储结构中, 以便随后传送到探测 平台的分析仪器中。

由于在岩石钻取采样时, 岩石破碎消耗功率比较大。 因此, 在原有钻机设计的基础上, 后期又新增加了取心电 机, 一起驱动钻具驱动装置钻进采样, 其原理样机模型如 图5 (b) 所示。

LSAS的操作简单, 很容易做到自主运动。首先, 采样 工具放置在目标上。通过压缩支撑锤钻系统的内部弹簧来 设置和保持预载荷。一旦就位, 电机以大约 $800 \mathrm{rpm}$ 的速度 旋转钻头, 钻头开始钻入目标表面。冲击作用有利于加强 钻具对岩石的破碎效果, 加快钻进速度。钻头每转三圈施 加一次锤击冲击, 锤击动作由同一马达驱动。钻头的锤击 动作通过凸轮从动件实现, 该凸轮从动件在其旋转时迫使 锤子向上, 压缩弹簧, 随后以非常快的速度释放存储的能 量, 使钻头向下运动。当材料脱离岩石时, 可以沿着钻头 上的凹槽向上运动, 并进入样品仓。

研究人员对LSAS进行了广泛的开发测试, 以评估初始 设计, 对设计进行微调并优化性能。获得的LSAS性能表征 如下表所示。由于担心设计可能不适合硬质岩石, 因此设 计了“重型”版LSAS, 但未组装。然而, 即使在玄武岩中, “基 本”版LSAS也被证明是够用的, 这是火星上最难钻进的岩石。

Honeybee Robotics公司为NASA Ames 80kg K-10巡航 器开发了一种称为冲击式动态锥形钻进系统。冲击式钻进 器的初步试验和现场测试在手动操作模式下进行, 如图6所 示。测试在压实 $\left(1.9 \mathrm{~g} / \mathrm{cm}^{3}\right)$ 的月壤中进行, 钻深为 $0.9 \mathrm{~m}$ 。 尽管速度不同, 所有的冲击式钻进器都能够达到底部, 速 度最快的在几十秒内达到最低点, 而最慢的时间需要3分钟。

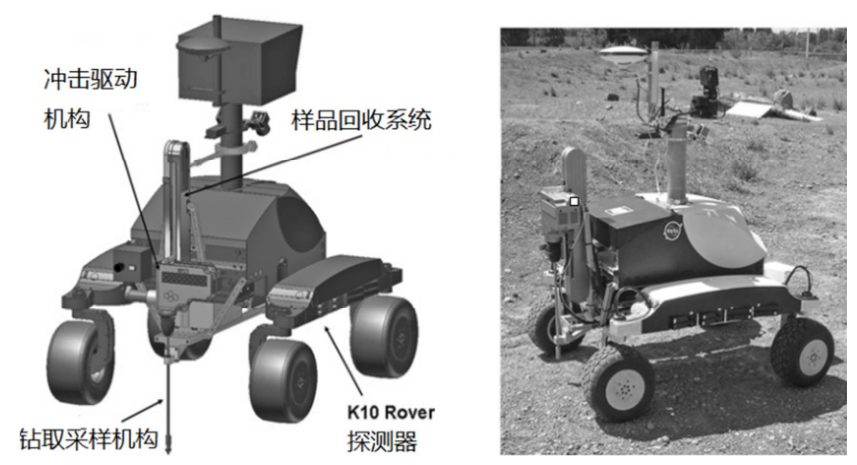

图6 Ames 80kg K-10巡航器采样器构型。
使用冲击式钻进器的另一个优点是钻进速度可用于评 估土壤性质。Honeybee Robotics的冲击式钻进器可以根据加 州承载比 ( $\mathrm{CBR}$ ) 评估土壤性质。 $\mathrm{CBR}$ 与土壤承载力和动 态模量（Huekelom and Klomp，1962）相关。承载力 $q$ 是在 浅基础设计中使用的参数, 动态模量 $(E)$ 或弹性模量 $(M r)$ 提供了在各种温度和应力状态下表征土壤的方法。因此, 从冲击式钻进器的钻进速度出发, 可以根据土壤的岩土性 质进一步评估月球特性, 并从这些得到月壤密度的估算值。 Honeybee Robotics公司最近为NASA Ames K-10巡航器建 立了一个完全自主的冲击钻进系统。该系统能够穿透高 $\mathrm{CBR}$ 土壤, 并实时测定土壤强度。

2008年, 欧空局ESA对在旋转钻进过程中实施持续地冲击 进行了研究, 希望以此提高对硬岩的钻进效率。ExoMars钻头 被认为是锤击机构钻 (DHM) 的经典之作。Galileo Avionica 与Helsinki University of Technology合作完成了这项研究。

机构描述：采用凸轮与压缩弹簧的组合, 弹簧可在凸 轮释放时提供冲击能量。凸轮释放冲击机构以后, 冲击击 机构会将冲击传递到钻具。通过连续的冲击作用使得待钻 岩石产生应力集中从而破碎硬岩。该机构适用于长度为 $505 \mathrm{~mm}$ 的钻杆。无螺旋的钻杆内径为 $24 \mathrm{~mm}$, 外径为 $27 \mathrm{~mm}$, 带螺旋的钻杆的外径为 $29 \mathrm{~mm}$, 用于切片提升。原型中使用 了Maxon EC22电机 (55W) 和直径为 $22 \mathrm{~mm}$ 的变速箱。

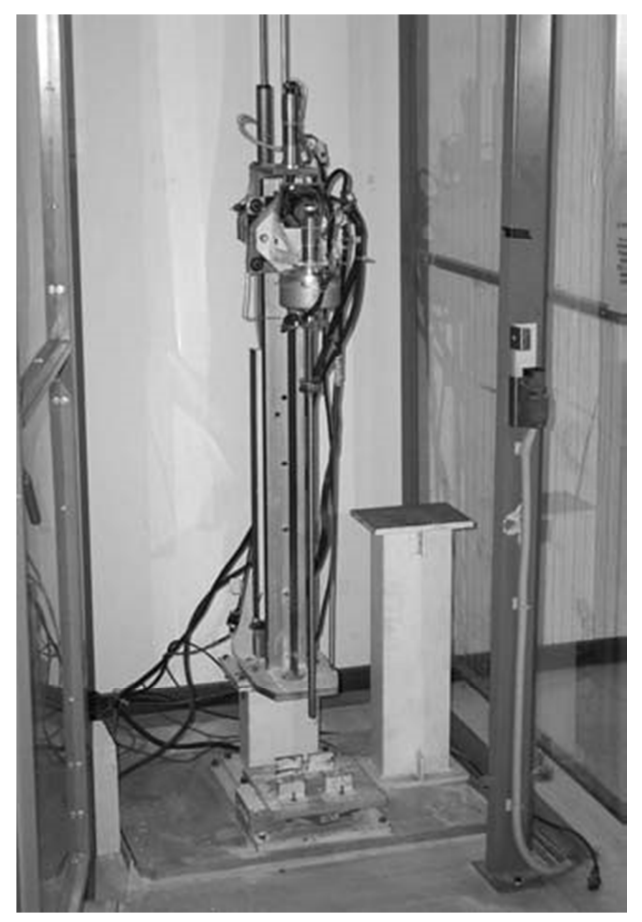

图7 ExoMars冲击钻进机构实验过程。

该原型机在不同类型的硬质材料和不同的工作条件下 进行了广泛的测试。测试中使用了一套钻探测试设备以及 一组专门设计的钻具, 如图7所示。测试结果表明, 该设备 可以在在花岗岩（压缩强度 $160 \mathrm{MPa}$ ）上以大约 $0.02 \mathrm{~mm} / \mathrm{min}$ 的速度进行钻取采样, 钻取时的钻压力较小 (100N), 功 率消耗非常低 (5-10W) [12]。

近年来, 欧空局ESA一直致力于水冰采样器研究。该水 冰采样器包括两个部分：钻取部分和定位部分 [19]。

其中钻取子系统的工作过程及原理如图8和图9所示。 


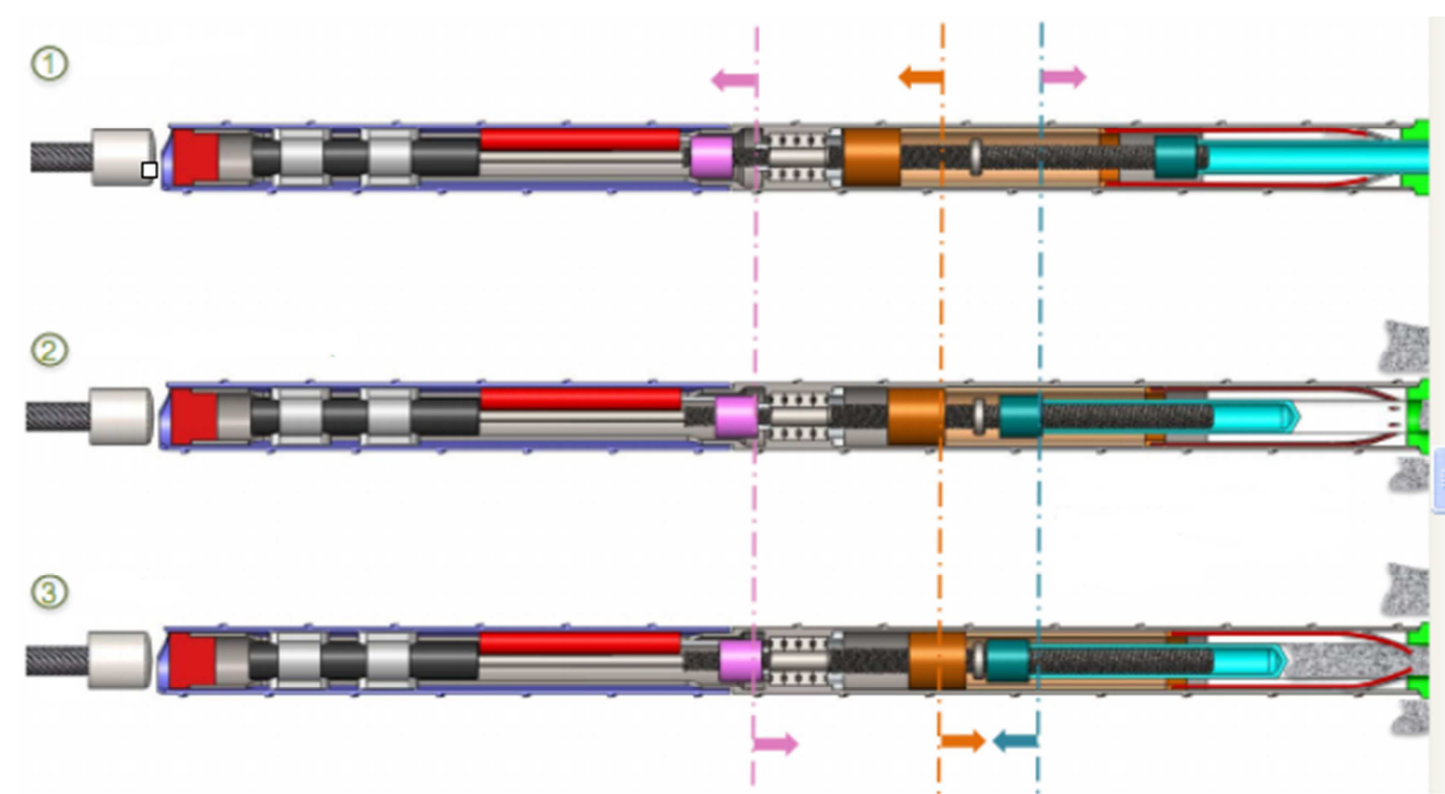

图8 钻取部分工作过程示意图。
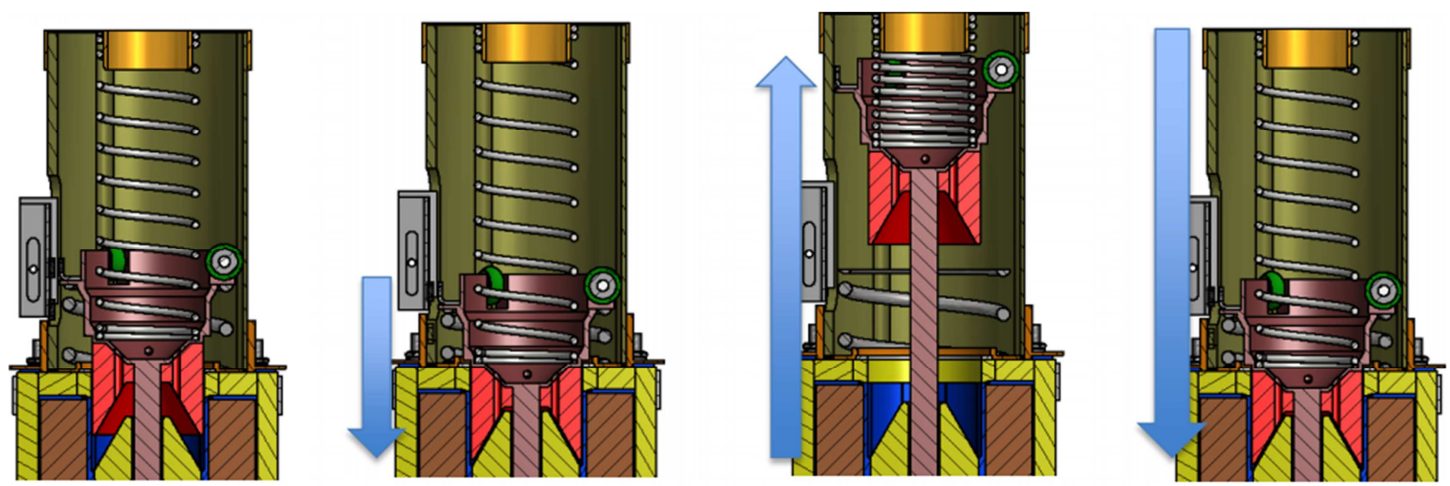

图9 冲击过程示意图。

冲击机构安装于芯轴组件的顶部; 冲击频率为 10-15赫 兹; 传递能量为 2 焦耳; 在共振模式下工作。

经过多次测试表明, 上述钻取设备具备钻进水冰的能 力, 钻进过程中的冲击对水冰的钻进有很大作用, 在冲击 力作用下，水冰易产生应力集中，从而发生破碎。

美国NASA及喷气推进实验室 (JPL) 和仿生机器人技 术实验室联合研制的CRUX钻取采样器是一种冲击回转式 钻取采样器, 该采样器应用于地外行星深层采样作业[20], 如图10所示。

CRUX钻取采样器由两个动力装置分别驱动, 能够实现 回转、冲击及回转冲击三种钻进模式。其采用单一钻杆的钻 进深度大于 $1 \mathrm{~m}$, 并可以通过增加钻杆的方式提高钻进深度。 CRUX钻取采样器总功率为 $180 \mathrm{~W}$, 其中旋转驱动功率为 $20 \mathrm{~W}$, 冲击驱动功率为 $160 \mathrm{~W}$; 旋转速度为 $100 \mathrm{r} / \mathrm{min}$; 单次冲击功 $2 \mathrm{~J}$; 冲击频率为每分钟 1700 次, 属于声频高频冲击激励; 进尺速 率为 $0.24 \mathrm{~cm} / \mathrm{min}$ 。当在地外行星表面进行深层采样时, 钻进 压力受限于探测器质量以及重力加速度。CRUX采样器通过 施加高频的冲击作用可以降低介质的抗破坏能力, 在轴向钻 压力很小的情况下加强钻取采样器对坚硬介质的破碎效果。
与普通旋转钻取采样器相比, 在相同的钻进压力下CRUX钻 取采样器能够获得更好的破碎效果。

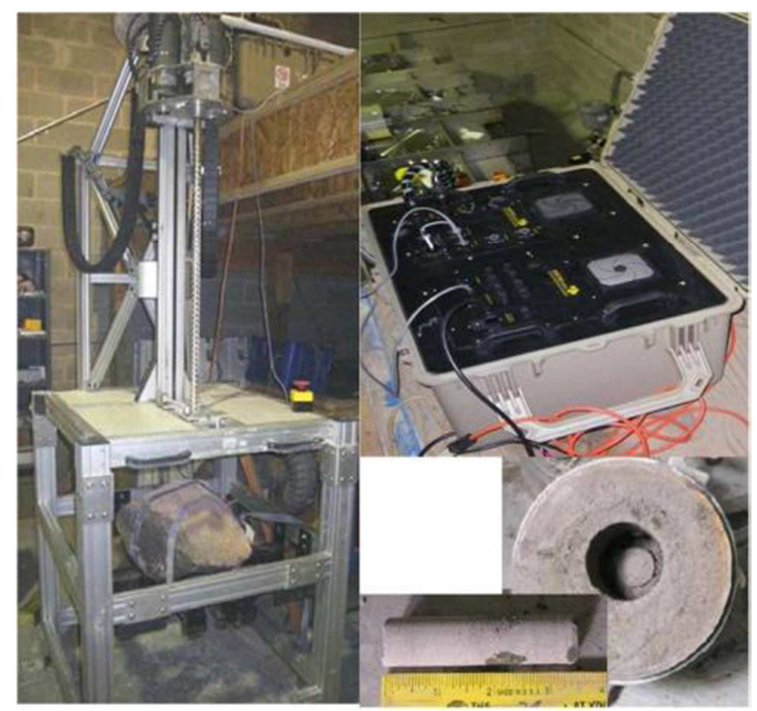

图10 CRUX钻取采样器及其控制箱。 


\section{4. 总结与展望}

对比国外星壤采样技术, 采样设备几乎均采用冲击或 冲击结合回转的工作模式, 并且常用弹簧凸轮式冲击机构 来施加冲击作用, 冲击能量以应力波的形式在机构中传递。 综合全球地外天体钻取采样先进经验, 在破碎岩石, 水冰 和其他星壤介质时, 冲击式采样法拥有以下几方面优势:

1. 冲击作用可以提高目标介质破碎效率。在冲击力作 用下, 介质易产生应力集中, 破碎形式多体现为破碎坑的 形式。破碎坑的形成有助于侵入设备破碎介质。因此冲击 作用能提高设备对高硬度岩石和星壤的破碎能力, 并增加 轴向潜入深度。

2. 在冲击作用下,可以利用惯性力代替钻压力,有效减 小破碎侵入过程中所需要的静压力和总能量,能够更好的适 应低重力低能耗条件下的采样工作。

3. 冲击产生的振动有利于侵入过程。振动一方面能减 小采样器与破碎对象之间的黏性阻力和孔底钻压力, 有效 减少卡钻、停钻现象的发生, 提高了可靠性（如luna20）; 另一方面, 振动使得碎屑松动, 更有利于排屑。

目前, 国内外对冲击系统的研究大部分只针对冲击功和 冲击频率等宏观参数对采样设备的破碎钻进过程进行了研 究。但在钻取采样过程中, 冲击载荷对岩石及土壤的破碎效 果, 以及冲击作用下被钻对象所获得的冲击能量和钻杆对冲 击能量的传递效率等科学问题未得到合理的理论解释、相关 仿真分析和实验的支持。对冲击机构本身的锤杆质量比、锤 杆刚度比、锤杆构型和应力波波形等设计参数对冲击能量在 钻杆中传递效率和对岩石破碎效果的影响进行的相关研究 也比较有限。因此, 未来我国需要在星壤钻取采样装置设计 阶段开展有关冲击作用的分析与研究工作, 并且应将相关研 究方向的重点放在冲击机构本身的参数优化设计工作上。

\section{5. 结束语}

针对关键技术攻关阶段尚未解决的问题和关键技术点, 需要开展深入研究工作。其中, 在钻取采样过程中, 冲击 载荷对月壤的破碎作用效果, 以及冲击作用下，钻头和被 钻对象所获得的冲击能量或钻杆对冲击能量的损耗效率等 研究问题均未得到合理的理论解释或相关试验数据的支持。 因此, 需要在钻取采样装置的设计阶段继续开展有关星壤 钻取采样冲击作用的分析与研究工作, 构建冲击载荷做用 下钻头切削岩石的理论模型以及钻杆在钻进过程中传递冲 击应力波能量的理论模型, 并对其进行仿真分析, 合理地 规划和设计冲击钻进试验, 用试验数据验证并支撑理论模 型的正确性, 为钻取采样装置的可靠钻进提供技术保障。

\section{致谢}

本文为国家自然科学基金一般项目《基于散体介质流 变理论的月壤与钻具作用机理研究》 (11502277)的阶段性成果 之一。

\section{参考文献}

[1] 姜生元, 张明, 张玉良等.宇航员手持式高频冲击采样装置的 设计研究 [J].深空探测学报,2015,6(2):131-136。

[2] 杨甘生, 冲击回转碎岩机理探究 [J].探矿工程(岩土钻掘工 程),2013,40(2):19-21。

[3] 刘德顺, 李夕兵, 朱萍玉.冲击机械动力学与反演设计 $[\mathrm{M}]$. 北京: 科学出版社, 2007 。

[4] 李夕兵, 赖海辉, 朱成忠. 冲击载荷下岩石破碎能耗及其力 学性质的探讨 [J] . 矿冶工程, 1988, 8(1): 15-19。

[5] 许金余，吕晓聪，张军，等. 围压条件下岩石循环冲击损伤 的能量特性研究 $[\mathrm{J}]$. 岩石力学与工程学报, 2010, 29(增 刊2): 4159-4165。

[6] 赵统武.冲击钻进动力学[M].冶金工业出版社,1996。

[7] 徐小荷, 余静. 岩石破碎学 [M]. 北京: 煤炭工业出版社, 1984: 6-157。

[8] Franca LFP. A bit-rock interaction model for rotary-percussive drilling[J]. International Journal of Rock Mechanics and Mining Sciences, 2011, 48(5): 827-835.

[9] Bar-Cohen, Y., Sherrit, S., Bao, X., Badescu, M.Aldrich, J. and Chang, Z. (2007) Ultrasonic/sonic driller/corer (USDC) as a subsurface sampler and sensors platform for planetary exploration applications. Proceedings of the NASA Science and Technology Conference (NSTC-07), University of Maryland Conference Center, 19-21 June 2007.

[10] Menon, C,Ayre, M. and Ellery, A. (2006a) Biomimetics, a new approach for space systems design. ESA Bulletin, (125), 20-26.

[11] OLSSONWA.The compressive strength of tuff as a function of strain rate from 10-6 to 103/sec[J]. International Journal of Rock Mechanics and Mining Sciences \& Geomechanics Abstracts, 1991, 28(1): 115-118.

[12] 滒泰宁,再恒谦,段新胜.宇宙探索与钻探技术[J].探矿工程(岩 土钻掘工程),2010,37(1):4。

[13] Osinski,G.R.,Lee,P.,Parnell,J, Spray, J.G. and Baron, M.(2005)A case study of impact induced hydrothermal activity:The Haughton impact structure, Devon Island, Canadian High Arctic. Meteoritics and Planetary Science, 40 (HMP Special Issue), 1859-1877.

[14] Final Report for Apollo Lunar Surface Drill(ALSD) [R], Martin Marietta Co-rporation, November 1, 1968: II -5 6.

[15] Zacny K, Wilson J, Chu P, et al. Prototype rotary percussive drill for the Mars Sample Returnmission[C]//Aerospace Conference, 2011 IEEE. IEEE, 2011: 1-8.

[16] Kriechbaum K, Brown K, Cady I, et al. Results from Testing of Two Rotary Percussive Drilling Systems[C]//Earth and Space 2010@s Engineering, Science, Construction, and Operations in Challenging Environments. ASCE, 2010.

[17] Gorevan, S.P.et al. (2003a) Rock Abrasion Tool: Mars Exploration Rover Mission[R],2003:Res.108. 
[18] Scott Stanley, Sean Dougherty, Jacques Laramee.The Low-force Sample Acquisition System[R]. Alliance Space systems, LLC.

[19] Ross Hironakaand Scott Stanley. Lightweight Low Force Rotary Percussive Coring Tool for Planetary Applications [C]. Proceedings of the 40th Aerospace Mechanisms Symposium, NASA Kennedy Space Center, 2010:17 30.
[20] Lobitz, B., Wood, B.L., Averner, M.A. and McKay, C.P. (2001) Use of spacecraft data to derive regions on Mars where liquid water would be stable. Proceedings of the National Academy of Sciences of the United States of America, 98, 2132-2137. 